https://helda.helsinki.fi

\title{
Do patients with Meniere's disease have attacks of syncope?
}

\section{Pyykko, Ilmari}

\section{7-10}

Pyykko, I, Manchaiah , V , Zou , J , Levo , H \& Kentala , E 2017 , ' Do patients with Meniere's disease have attacks of syncope?' ' Journal of Neurology , vol. 264 , pp. S48-S54 . https://doi.org/10.1007/s00415-017-8452-9

http://hdl.handle.net/10138/298092

https://doi.org/10.1007/s00415-017-8452-9

publishedVersion

Downloaded from Helda, University of Helsinki institutional repository.

This is an electronic reprint of the original article.

This reprint may differ from the original in pagination and typographic detail.

Please cite the original version. 


\title{
Do patients with Ménière's disease have attacks of syncope?
}

\author{
Ilmari Pyykkö ${ }^{1}$ Vinaya Manchaiah ${ }^{2,3,4,5} \cdot$ Jing Zou $^{1,6} \cdot$ Hilla Levo $^{7} \cdot$ \\ Erna Kentala ${ }^{7}$
}

Received: 29 December 2016/Revised: 6 March 2017/Accepted: 10 March 2017/Published online: 20 March 2017

(C) Springer-Verlag Berlin Heidelberg 2017

\begin{abstract}
The aim of the present study was to evaluate the prevalence and associated factors for syncope among patients with Ménière's disease (MD). An attack of syncope was defined as a sudden and transient loss of consciousness, which subsides spontaneously and without a localizing neurological deficit. The study used an acrosssectional survey design. Information from a database consisting of 961 individuals was collected from the Finnish Ménière Association. The data contained case histories, general health-related quality of life (HRQoL), and impact measurements of the complaints. In the current
\end{abstract}

This manuscript is part of a supplement sponsored by the German Federal Ministry of Education and Research within the funding initiative for integrated research and treatment centers.

Ilmari Pyykkö

ilmari.pyykko@uta.fi

1 Hearing and Balance Research Unit, Department of Otolaryngology, University of Tampere, Tampere, Finland

2 Department of Speech and Hearing Sciences, Lamar University, Beaumont, TX, USA

3 Department of Behavioral Science and Learning, Linnaeus Centre HEAD, Swedish Institute for Disability Research, Linköping University, Linköping, Sweden

4 Audiology India, Mysore, Karnataka, India

5 Department of Speech and Hearing, School of Allied Health Sciences, Manipal University, Karnataka, India

6 Department of Otolaryngology-Head and Neck Surgery, Center for Otolaryngology-Head and Neck Surgery of Chinese PLA, Changhai Hospital, Second Military Medical University, Shanghai, China

7 Department of Otolaryngology, University of Helsinki, Helsinki, Finland study sample, syncope occurred in $12.3 \%$ of the patients with MD. It was more prevalent among elderly persons and among those with a longer duration of MD. Syncope was significantly associated with disturbances of otolith function reflected as Tumarkin attacks, gait and balance problems, environmental change of pressure, and physical strain. It was also associated with visual blurring; in fact, patients with otolith dysfunction in MD often experience visual field changes. It was also associated with headache, but not with migraine. Syncope was experienced as frightening and HRQoL was significantly worsened. The patient had higher anxiety scores, and suffered more from fatigue. The results demonstrate that neurally mediated syncope occurs in patients with an advanced form of MD who suffer from Tumarkin attacks due to failure in otolith function. The mechanism seems to be triggered through the vestibular sympathetic reflex when the otolith system fails due to disrupted utricular otolithic membrane mediate erroneous positional information from the otolith organ to the vasomotor centres in the brain stem and medulla.

Keywords Ménière's disease · Tumarkin attacks · Drop attack · Fainting vestibular disorder - Otolith organ · Syncope

\section{Introduction}

Transient loss of consciousness (T-LoC) is a term that encompasses all disorders characterized by self-limited loss of consciousness, irrespective of mechanism. The position paper of The European Heart Rhythm Association (EHRA) defines "syncope" as a transient loss of consciousness due to transient global cerebral hypoperfusion, and is characterized by rapid onset, short duration, and 
spontaneous complete recovery [1]. Despite intensive investigations, about $50 \%$ of syncope patients entering the emergency unit will have no specific disorder and will be diagnosed with vasovagal syncope. Most effort is put in cardiovascular function evaluations, and commonly, the neurological causes of syncope have been excluded [2-4]. However, most syncope units recommend that a neurologist be included in the unit's syncope team to assist in diagnosis of the patients [1]. The factors found to be associated with non-cardiac syncope such as age $<40$ years, a long prodrome, nausea, diaphoresis, and blurred vision all resonate strongly with the demographic and clinical features of vasovagal syncope [5]. In only one report has an otological reason for syncope been detected. In their study with elderly patients, O'Mahony and Foote demonstrated that about $2 \%$ of syncope that they classified as unexplained syncope was caused by a vestibular disorder [6].

Recent experiments on space flights confirm that the inner ear otolithic input is an important regulator of blood pressure and heart rate in humans [7] and leads to orthostatic dizziness and also syncope due to a sudden drop in blood pressure. The otoliths detect a position change with respect to gravity within milliseconds, and play a key role in the activation of the vascular tone through the vestibular sympathetic reflex [8]. In an experimental animal model, researchers demonstrated that the otolith input influenced mechanisms regulating blood pressure and heart rate, and seemed to be one of the key operators in regulating the homeostatic control of blood pressure during stance [9]. The anatomical connection of the vestibular sympathetic reflex pathway has been described in animals [10]. The activated cells in this pathway were concentrated in the caudal inferior and medial vestibular nuclei, otolith-recipient regions, and sent axonal projections to the rostral and caudal ventrolateral medullary areas [10]. These regions are integral parts of the sympathetic pathway to the spinal cord, ultimately leading to activation of the blood vessels and controlling the heart rate [11]. The vestibular sympathetic reflex is thought to be particularly important in the prevention of a drop in blood pressure and orthostatic intolerance because the vestibular sympathetic reflex can be elicited at the first sensing of motion [12].

$\mathrm{MD}$ is a chronic disorder in that patients complain of vertigo, hearing loss, tinnitus, gait problems, postural instability, and in a severe form, patients suffer from a sudden loss of balance often referred to as "drop attacks" or "Tumarkin attacks" [13]. In almost all patients with MD, an endolymphatic hydrops can be demonstrated [14]. Calzada et al. [15] evaluated the otolithic membrane in patients with endolymphatic hydrops and Tumarkin attacks undergoing ablative labyrinthectomy. All patients with vestibular drop attacks showed evidence of a disrupted utricular otolithic membrane. During the attacks, patients experience a sudden loss of balance that occurs without warning, commonly without loss of consciousness. The patients typically report the sensation of being pushed, and they fall usually in the same direction with repeated falls. In some cases, patients report a sudden tilt of the environment simultaneous with the fall [16]. The pathophysiology of Tumarkin attacks has been linked to sudden changes in the otolith function of the utricle or saccule due to pressure gradients within the inner ear, which is also linked to abnormal ocular tilt function [17].

So far, there are no published reports that establish a link between Tumarkin attacks and T-LoC, more specifically syncope. During the clinical interactions, we have encountered several cases that had MD and also experienced syncope; detailed investigations suggested that the attacks were associated with Tumarkin attacks [18]. In the current study, we further investigated the prevalence and associated factors for syncope among patients with MD. Specifically, we searched for evidence that would support the association of Tumarkin attacks and attacks with syncope.

\section{Method}

\section{Study design and participants}

The study used a cross-sectional survey design. Permission was obtained from the Finnish MénièreFederation (FMF; Suomen Meniere-liitto) to contact their members, asking them to complete an extensive questionnaire on symptoms related to MD. Under Finnish law, this kind of study does not need ethical approval. From 1646 members, the data was collected with a written questionnaire (740 of 1200 responded with response rate of $61.6 \%$ ) or with an Internetbased questionnaire (176 of 466 responded with response rate of $37.7 \%$ ). Responses to both the written questionnaire and the Internet-based questionnaire were identical. Of the total 961 responses, missing data was noticed in 45 questionnaires, which resulted in a usable sample of 916 participants. Of the samples included in the analysis composed of 916 individuals, 658 were females and 258 were males. The mean age was 60.6 years (SD 13.0 years). Their MDspecific symptoms had lasted an average of 9.8 years (SD 5.1 years). Syncope was defined as a sudden and transient loss of consciousness, which subsides spontaneously and without localizing neurological deficit. The question regarding syncope was included in the questionnaire in 
connection with "neurological complaints." The question was formulated as "Do you have or have had attacks during which you have lost consciousness?" to differentiate the origin of syncope to neurological causes (versus other conditions), we had also asked participants to declare their extended neurological complaints, diagnosis history, and other health conditions including concussion, epileptic seizures, psychiatric and degenerative disorders, among others.

\section{Data collection using questionnaires}

A detailed 86-item otoneurology questionnaire was used for assessing the symptoms and consequences of the disorder [19]. The EuroQol EQ-5D-3L tool was used to study the general health-related quality of life (HRQoL). The EQ-5D-3L tool has a test-retest reliability of 0.66 , and has validity in relation to other generic HRQoL measures. A disease-specific impact assessment was done using a combination of open-ended and closed questions. The questionnaire based on the International Classification of Functioning Disability and Health (ICF) was used to classify the impact of the disease at the individual level.

\section{Data analysis}

Non-parametric tests such as the Mann-Whitney $U$ test and the Kruskal-Wallis $H$ test were used to explore the data to investigate the association between syncope and demographic details, as well as complaints about the disease. The dependencies between these factors were examined using the logistic regression analysis. A $p$ value of 0.05 was used for interpretation of statistical significance.

\section{Results}

1. Association between syncope, age, and duration of the disease

Attacks of fainting leading to syncope occurred in 113 out of 916 participants (i.e., 11.3\%). Figure 1 presents the mean and standard error of mean (SEM) indicating the prevalence of syncope among patients with MD, classified by different age groups and duration of vertigo. The attacks of syncope tended to be more frequent among elderly patients than in young patients (Mann-Whitney $U=21,700.5, p=0.007$ ) and in those who had a longer duration of MD (MannWhitney $U=36,997.5, p=0.001$ ).

2. Association of vestibular complaints with syncope

The Kruskal-Wallis $H$ test was performed to study the association between the attacks of syncope and the complaints regarding MD, which indicated that the association of syncope was mostly related to faulty otolith function (see Table 1). The main association was found in the items: Tumarkin attacks, a tendency to fall, postural instability, movement instability, and vertigo induced by head movement. These all complaints originate in malfunction of the otolith system. The rotational vertigo attacks were inversely correlated with syncope. Interestingly, a change of environmental pressure and vertigo induced by physical strain were also associated with syncope, indicating that these were provoking items for the attacks.
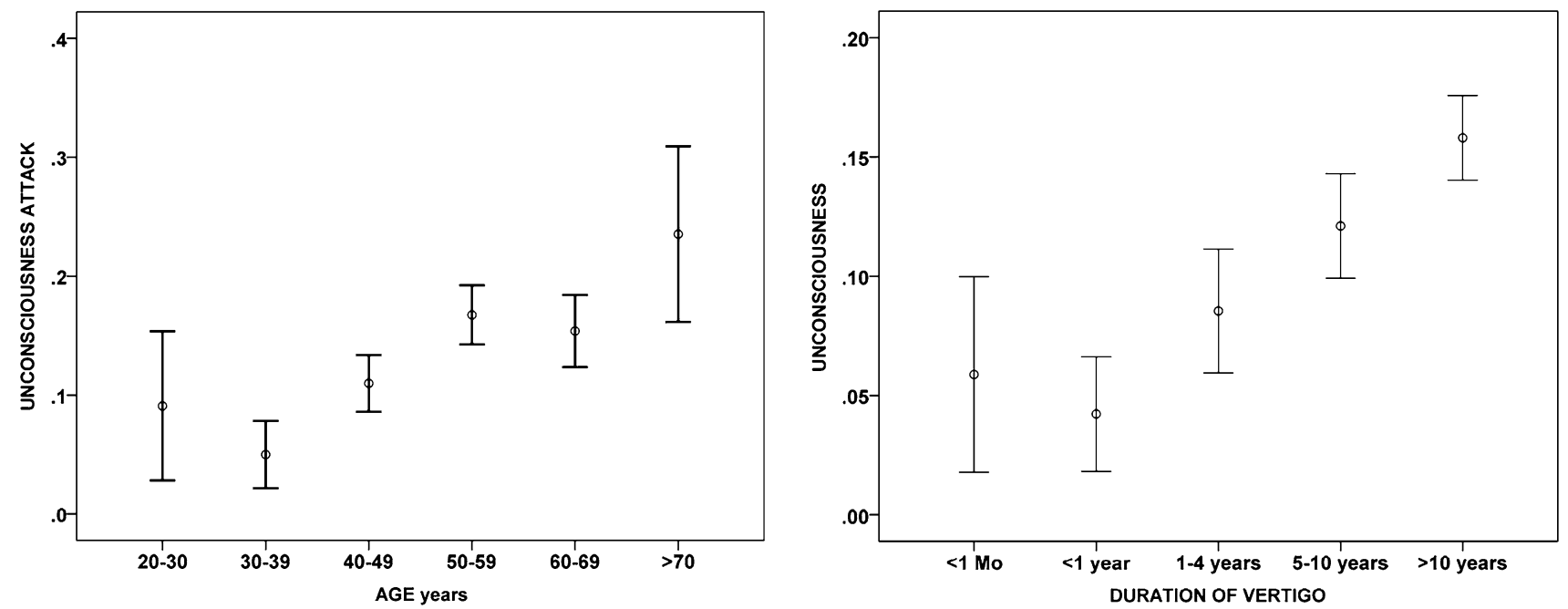

Fig. 1 Prevalence (means and SEM) of syncope among patients with Ménière's disease classified into different age groups (left) and different duration of the disease (right) 
Table 1 Association of vestibular complaints and provoking factors with syncope as indicated by the KruskalWallis $H$ test

\begin{tabular}{lccc}
\hline Complaint & Chi-square & $d f$ & Sig. ( $p$ value) \\
\hline Tumarkin attacks & 16.388 & 1 & $\leq 0.0001$ \\
Tendency to fall & 16.263 & 1 & $\leq 0.0001$ \\
Postural instability & 24.456 & 1 & $\leq 0.0001$ \\
Movement instability & 11.988 & 1 & 0.001 \\
Head position change induced vertigo & 7.513 & 1 & 0.006 \\
Rotational vertigo attacks & 5.621 & 1 & 0.022 \\
Environmental pressure change induced vertigo & 27.698 & 1 & $\leq 0.0001$ \\
Physical strain induced vertigo & 13.485 & 1 & $\leq 0.0001$ \\
\hline
\end{tabular}

Table 2 Association of neurological complaints with attacks of syncope as indicated by the Kruskal-Wallis $H$ test

\begin{tabular}{lccc}
\hline Complaint/disorder & Chi-square & $d f$ & Sig. ( $p$ value) \\
\hline Headache during the attack & 4.709 & 1 & 0.030 \\
Headache outside of vertigo attack & 0.632 & 1 & 0.430 \\
Migraine & 3.024 & 1 & 0.098 \\
Blurring of vision & 4.777 & 1 & 0.030 \\
Visual field changes & 10.825 & 1 & 0.001 \\
Speech problems during the attack & 21.028 & 1 & $\leq 0.0001$ \\
Facial numbness & 4.110 & 1 & 0.049 \\
Ischemic heart disease & 4.552 & 1 & 0.044 \\
Ischemic brain disease & 14.184 & 1 & $\leq 0.0001$ \\
Thyroid gland problems & 5.712 & 1 & 0.019 \\
History of brain concussion & 11.845 & 1 & 0.001 \\
Kidney disease & 4.231 & 1 & 0.075 \\
\hline
\end{tabular}

Furthermore, we evaluated whether different neurological complaints or diseases affecting other organs would be related to attacks of syncope. Attacks of syncope were associated with headaches during the attack, and also with blurring of vision, speech problems, visual field changes (i.e., absence of visual field, visual neglect), numbness of the face, ischemic heart and brain disease, thyroid problems, and previous brain concussion (see Table 2). However, it is noteworthy that migraines, diabetes and kidney disorders were not significantly associated with attacks of syncope.

Several diseases could interplay with attacks of syncope. In patients who had suffered a brain concussion, 99 participants (i.e., 10.3\%) reported to have had syncope. 65 participants out of 892 (i.e., 7.3\%) had suffered from cerebrovascular insult and could have syncope. The kidney insufficiency was present only among 9 participants out of 887 patients (i.e., $1 \%$ ), but could be a predictor of syncope. Finally, 109 participants had thyroid problems that were associated with syncope.

In logistic regression analysis, an attack of syncope was related to the frequency of the intensity of postural instability, a tendency to fall in attacks of MD, presence of a brain concussion, absences of rotatory vertigo attacks, and anxiety (see Table 3). Interestingly, migraines were not included in the model. A history of brain concussion and ischemic brain disease were included in the model. The model was statistically significant $(p<0.0001)$, and could explain $13.0 \%$ of the variability in the model.

\section{Consequences of syncope on well-being}

Finally, we evaluated the relationship between attacks of syncope, and variables such as physical and psychological

Table 4 Impact of syncope on patents with Ménière's disease

\begin{tabular}{lccc}
\hline & Chi-square & $d f$ & Sig. $(p$ value $)$ \\
\hline EQoL-VAS & 15.019 & 1 & $\leq 0.0001$ \\
EQoL-5D & 16.965 & 1 & $\leq 0.0001$ \\
MD severity rating & 7.153 & 1 & 0.007 \\
Anxiety & 15.581 & 1 & $\leq 0.0001$ \\
Energy & 8.626 & 1 & 0.001 \\
\hline
\end{tabular}

Table 3 Outcome of logistic regression analysis on items explaining syncope in patients with Ménière's disease

\begin{tabular}{lrlcccc}
\hline & \multicolumn{1}{c}{$B$} & SE & Wald & $d f$ & Sig. & $\operatorname{Exp}(B)$ \\
\hline Constant & -2.937 & 0.295 & 99.216 & 1 & $\leq 0.0001$ & 0.053 \\
Intensity of instability & 0.270 & 0.127 & 4.488 & 1 & 0.034 & 1.310 \\
Tendency to fall & 0.921 & 0.244 & 14.230 & 1 & $\leq 0.0001$ & 2.511 \\
Anxiety & 0.269 & 0.109 & 6.111 & 1 & 0.013 & 1.309 \\
Brain concussion & 0.873 & 0.315 & 7.673 & 1 & 0.006 & 2.394 \\
Rotational vertigo & -0.633 & 0.241 & 6.909 & 1 & 0.009 & 0.531 \\
Ischemic brain disease & 0.869 & 0.354 & 6.004 & 1 & 0.014 & 2.384 \\
\hline
\end{tabular}


consequences (i.e., anxiety) experienced by patients with MD, self-rated severity ratings of MD, and generic HRQoL using the Kruskal-Wallis $H$ test. The patients with attacks of syncope had worsened HRQoL, experienced a more severe impact of MD, and had increased anxiety and fatigue (see Table 4).

\section{Discussion}

The current study was aimed at investigating the possible existence of syncope among patients with MD. Syncope is not an uncommon phenomenon in our society, and up to $20 \%$ of people under the age of 75 have experienced it [12]. Due to the high incidence of syncope in the general population, we had anticipated that nearly one third of the study participants might report syncope. However, only $12.3 \%$ of MD patients in the current study reported syncope, and that the syncope was associated with Tumarkin attacks and provoking factors which caused the attacks. Although we do not understand the reason for this low prevalence, we anticipate that this may be related to participants not remembering the episodes of T-LoC. Radtke et al. [20] evaluated syncope and orthostatic disorders among patients with dizziness and vertigo, and reported a lifetime prevalence of $12.5 \%$, although no specific details about the vestibular disorders were provided in this study. In our previous work, we reported that $4 \%$ of the patients claimed that syncope occurred in connection with vertigo attack [18]. Thus, syncope associated with MD is less than the prevalence of syncope observed in this study (i.e., $12.3 \%$ ), and may be linked to other reasons leading to syncope [18]. Overall, our findings show that patients with MD have syncope, which has been linked to Tumarkin attacks, indicating that the otolith organ is involved in this complaint process.

Our findings confirm the results from animal experiments that impairment of the otolith system can lead to a drop in blood pressure and syncope [21]. In experimental animals, sinusoidal galvanic vestibular stimulation (sGVS) led to changes in peripheral resistance and reduced cardiac output and further resulted in hypotension, bradycardia, and decreased cerebral blood flow [22, 23]. In some animals, sGVS produced vasovagal syncope-like symptoms, including fatigue and uncoordinated movements or spontaneous falling [11]. The authors further suggested that the activity responsible for the vasovagal oscillations arises in low frequency and involves otolith neurons with orientation vectors close to the vertical axis of the head. These neurons are likely to provide critical input to the vestibulosympathetic reflex to increase blood pressure and heart rate upon changes in the head position relative to gravity, and contributes to the production of vasovagal oscillations, vasovagal responses, and syncope when the baroreflex is inactivated. Stimulation of labyrinthine receptors has been demonstrated to alter the firing of vasoconstrictor efferents of the sympathetic nervous system [24], and to modify blood flow through the arterial vascular beds [25], suggesting that vestibular influences on the sympathetic nervous system serve to maintain stable blood pressure during alterations in posture. The syncope attacks in experimental animals were very much the same as the neurogenically mediated vasovagal syncope [26] occurring in association with Tumarkin attacks. We hypothesize in line with Raphan et al. that the vestibular sympathetic reflex interferes with the medullary rostral and caudal lateral medullary nucleus and interferes with the function of the vasoregulatory center and causes syncope [27]. The output would cause vasovagal attack with reduced muscle sympathetic vessel tonus with simultaneous paradoxical bradycardia, leading to falls and also syncope for a variable time [28].

In a statistical work-up, we found out that environmental pressure changes and physical strain were associated with attacks of syncope. These items seemed to provoke attacks of Tumarkin associated with syncope, indicating susceptibility of the otolith organ for any pressure change in MD. Recently it has been documented in MRI that all patients with MD have endolymphatic hydrops [14]. Calzada et al. [29] evaluated the otolithic membrane in patients with endolymphatic hydrops and vestibular drop attacks undergoing ablative labyrinthectomy. All patients with vestibular drop attacks showed evidence of a disrupted utricular otolithic membrane, whereas only 50 and $56 \%$ of patients with delayed endolymphatic hydrops and $\mathrm{MD}$, respectively, demonstrated otolithic membrane disruption. Thus, endolymphatic hydrops seems to make the patients liable for even small pressure changes, indicating that the underlying pathophysiology in sudden drop attack results from injury to the otolithic membrane of the saccule and utricle. We hypothesize that a sudden change in environmental pressure would lead to pressure changes in the endolymph/ perilymph system and distort the otolith membrane, provoking a Tumarkin attack and eventually leading to syncope. We also found that syncope was negatively correlated with rotatory vertigo attacks, indicating that the role of semicircular canals may not be important, confirming the findings in animals $[27,30]$. Our findings indicate that the main reason for syncope in patients with MD is the malfunctioning otolith organ, which disturbs the vestibular sympathetic reflex originating from the otoliths, thus provoking the paradoxical vasovagal attack leading to syncope.

In the present study, disorders associated with syncope were ischemic cerebro-vascular disease and cardiovascular diseases. We suggest that associated vascular complaints, (e.g., cardio-vascular disorders), may prove to be 
susceptibility factors rather than causes of syncope. The etiology of drop attacks is liable to be misdiagnosed even if the symptoms and signs were consistent with MD. Hence, these confounders were included in the logistic regression analysis, and were able to demonstrate that the vestibular component still contributed to syncope attacks.

The consequences of syncope in MD are severe. Tumarkin attacks and syncope are frightening. In the present study, the HRQoLwas significantly worsened among patients with syncope. The patient had higher anxiety scores and experienced more fatigue. Loss of vitality has also been reported as a consequence in patients with orthostatic disorder [8]. The therapeutic possibilities seem to be limited. Gentamicin therapy is the most common, and provides relieve from Tumarkin attacks in $60-90 \%$ of patients [31]. However, some patients with Tumarkin attacks are quite resistant against therapy [32]. Cardio-specific beta-blockers may be beneficial as they inhibit the vestibular sympathetic cardioinhibitory reflex as has been suggested [33].

\section{Strengths and limitations}

While the study, we believe is the first of its kind in the MD population, it has a number of limitations and the results have to be viewed with caution. First, the attacks of syncope were not controlled for eyewitnesses. As patients tend not to recall short lasting syncope due to post syncopal amnesia, the numbers of patients with this condition are probably greater than we reported here. Second, in routine clinical practice using case histories alone, it can be difficult to ascertain that patients lost consciousness, as they not always understand what this involves. Third, while we believe the majority of participants who reported T-LoC is because of syncope, it is difficult to completely exclude other possible causes of T-LoC (e.g., epileptic or psychogenic problems) using self-reported questionnaires alone. Fourth, the main weakness in this study was that the database did not specify whether the syncope was specifically linked to Tumarkin attacks. Therefore, the study contained confounders that may have interplayed with the mechanisms leading to syncope. Fifth, the main weakness of the study was not having a control group, although in our recent study we have compared MD patients who experience syncope with and without Tumarkin attacks [18]. Sixth, the otoneurological questionnaire has not been validated, although it has been used in many previous studies [19]. Finally, we also did not evaluate the otolith function among MD patients using VEMPs. It may be so that VEMP is sensitive, and could also in clinical cases point out which one of the subjects with Tumarkin attacks are in risk of syncope. It may be so that utricular otolithic input rather than a saccular one could actually impact the baroreceptor sensitivity $[34,35]$. In this case, the ocular VEMP could be useful as a predictor of possible risk. Hence, further research with a robust study design is necessary to investigate the association of Tumarkin attack with eye-witnessed syncope, and to study the characteristics of Tumarkin attacks linked with syncope.

\section{Conclusions}

Recent experiments in humans and in experimental animals indicate that the otolithic input is an important regulator of blood pressure and heart rate, and seems to be one of the key operators in the homeostatic control of blood pressure. In MD with endolymphatic hydrops, abnormal stimulation of the vestibular system could lead to orthostatic dizziness and even syncope due to a sudden drop in blood pressure. MD patients who experience Tumarkin attacks have sudden attacks of imbalance that occur without warning, and without any concomitant neurologic sequelae. In severe form, the patients with MD can have syncope with falls resulting in a complete lying position. Among patients with MD who have Tumarkin attacks, gait and postural disorders were associated with syncope. A fall in patients with MD was experienced as frightening and reduced the general HRQoL, causing anxiousness and fatigue. We recommend that health care personnel taking care of patients with MD should monitor the Tumarkin attacks and syncope. We also recommend active treatment for Tumarkin attack associated falls and syncope.

\section{Compliance with ethical standards}

Conflicts of interest None to declare.

\section{References}

1. Kenny RA, Brignole M, Dan GA, Deharo JC, van Dijk JG, Doherty C, Hamdan M, Moya A, Parry SW, Sutton R, Ungar A, Wieling W (2015) Syncope Unit: rationale and requirement-the European Heart Rhythm Association position statement endorsed by the Heart Rhythm Society. Europace 17(9):1325-1340. doi:10. 1093/europace/euv115

2. Thiruganasambandamoorthy V, Sheldon R (2015) Syncope confusion. CMAJ 187(7):521. doi:10.1503/cmaj.115-0030

3. Sheldon RS, Morillo CA, Krahn AD et al (2011) Standardized approaches to the investigation of syncope: Canadian Cardiovascular Society position paper. Can J Cardiol 27:246-253

4. Moya A, Sutton R, Ammirati F et al (2009) Guidelines for the diagnosis and management of syncope. Eur Heart J 30:2631-2671

5. Berecki-Gisolf J, Sheldon A, Wieling W, van Dijk N, Costantino G, Furlan R, Shen WK, Sheldon R (2013) Identifying cardiac syncope based on clinical history: a literature-based model tested in four independent datasets. PLoS One 8(9):e75255. doi:10.1371/journal.pone.0075255 (eCollection 2013)

6. O'Mahony D, Foote C (1998) Prospective evaluation of unexplained syncope, dizziness, and falls among community-dwelling elderly adults. J Gerontol A BiolSci Med Sci 53(6):M435-M440 
7. Hallgren E, Migeotte PF, Kornilova L, Delière Q, Fransen E, Glukhikh D, Moore ST, Clément G, Diedrich A, MacDougall H, Wuyts FL (2015) Dysfunctional vestibular system causes a blood pressure drop in astronauts returning from space. SciRep 5:17627. doi:10.1038/srep17627

8. Raphan T, Cohen B, Xiang Y, Yakushin SB (2016) A model of blood pressure, heart rate, and vaso-vagal responses produced by vestibulo-sympathetic activation. Front Neurosci 10:96. doi:10. 3389/fnins.2016.00096 PMID: 27065779

9. Yates BJ, Bolton PS, Macefield VG (2014) Vestibulo-sympathetic responses. Compr Physiol 4:851-887

10. Holstein GR, Friedrich VL Jr, Martinelli GP (2014) Projection neurons of the vestibulo-sympathetic reflex pathway. J Comp Neurol 522(9):2053-2074. doi:10.1002/cne.23517

11. McBride DW, Reis C, Frank E, Klebe DW, Zhang JH, Applegate $\mathrm{R}$ 2nd, Tang J (2016) An experimental model of vasovagal syncope induces cerebral hypoperfusion and fainting-like behavior in awake rats. PLoS One 11(9):e0163280. doi:10.1371/ journal.pone.0163280

12. Kaufmann H, Biaggioni I, Voustianiouk A, Diedrich A, Costa F, Clarke R et al (2002) Vestibular control of sympathetic activity. An otolith-sympathetic reflex in humans. Exp Brain Res 143:463-469. doi:10.1007/s00221-002-1002-3

13. Kentala E, Havia M, Pyykkö I (2001) Short lasting drop attacks in Meniere's disease. Otolaryngol Head Neck Surg 124:526-530

14. Pyykkö I, Nakashima T, Yoshida Y, Zou J, Naganawa S (2013) Meniere's disease: a reappraisal supported by a variable latency of symptoms and the MRI visualisation of endolymphatic hydrops. BMJ Open. doi:10.1136/bmjopen-2012-001555 (pii: e001555)

15. Calzada AP, Lopez IA, Ishiyama G, Ishiyama A (2012) Otolithic membrane damage in patients with endolymphatichydrops and drop attacks. OtolNeurotol 33(9):1593-1598. doi:10.1097/MAO. 0b013e318271c48b

16. Ishiyama G, Ishiyam A, Baloh RW (2003) Drop attacks and vertigo secondary to a non-meniere otologic cause. Arch Neurol 60:71-75

17. Brandt T, Dieterich M (1993) Vestibular falls. J Vestib Res 3(1):3-14

18. Pyykkö I, Manchaiah V, Zou J, Levo H, Kentala E (2017) Vestibular syncope: a disorder associated with drop attack in Ménière's disease. AurisNasus Larynx (Accepted)

19. Kentala E (1996) Characteristics of six otologic diseases involving vertigo. Am J Otol 17(6):883-892

20. Radtke A, Lempert T, von Brevern M, Feldmann M, Lezius F, Neuhauser H (2011) Prevalence and complications of orthostatic dizziness in the general population. ClinAuton Res 21(3):161-168. doi:10.1007/s10286-010-0114-2

21. Rose KM, Eigenbrodt ML, Biga RL et al (2006) Orthostatic hypotension predicts mortality in middle-aged adults: the
Atherosclerosis Risk In Communities (ARIC) Study. Circulation 114:630-636

22. Cohen B, Yakushin SB, Holstein BR (2011) What does galvanic vestibular stimulation actually activate? Front Neurol 2:90. doi:10.3389/fneur.2011.00090

23. Yakushin SB, Martinelli GP, Raphan T, Xiang Y, Holstein GR, Cohen B (2014) Vasovagal oscillations and vasovagal responses produced by the vestibulo-sympathetic reflex in the rat. Front Neurol 5:37. doi:10.3389/fneur.2014.00037

24. Kerman IA, Emanuel BA, Yates BJ (2000) Vestibular stimulation leads to distinct hemodynamic patterning. Am J Physiol Regul Integr Comp Physiol 279:R118-R125

25. Kerman IA, Yates BJ, McAllen RM (2000) Anatomic patterning in the expression of vestibulosympathetic reflexes. Am J Physiol Regul Integr Comp Physiol 279:R109-R117

26. Lewis T (1932) Vasovagal syncope and the carotid sinus mechanism. Br Med J 3723:873-876. doi:10.1136/bmj.1.3723.873

27. Raphan T, Cohen B, Xiang Y, Yakushin SB (2016) A model of blood pressure, heart rate, and vaso-vagal responses produced by vestibulo-sympathetic activation. Front Neurol 10:96. doi:10. 3389/fnins.2016.00096

28. Nowak L, Nowak FG, Janko S, Dorwarth U, Hoffmann E, Botzenhardt F (2007) Investigation of various types of neurocardiogenic response to head-up tilting by extended hemodynamic and neurohumoral monitoring. Pacing ClinElectrophysiol 30(5):623-630

29. Yates BJ (1996) Vestibular influences on the autonomic nervous system. Ann NY AcadSci 781:458-473

30. Xerri C, Barthélémy J, Harlay F, Borel L, Lacour M (1987) Neuronal coding of linear motion in the vestibular nuclei of the alert cat. I. Response characteristics to vertical otolith stimulation. Exp Brain Res 65:569-581. doi:10.1007/BF00235980

31. Viana LM, Bahmad F Jr, Rauch SD (2014) Intratympanic gentamicin as a treatment for drop attacks in patients with Meniere's disease. Laryngoscope 124(9):2151-2154. doi:10.1002/lary. 24716

32. Pyykkö I, Ishizaki H, Kaasinen S, Aalto H (1994) Intratympanic gentamicin in bilateral Meniere's disease. Otolaryngol Head Neck Surg 110:162-167

33. Fedorowski A, Melander O (2013) Syndromes of orthostatic intolerance: a hidden danger. J Intern Med 273(4):322-335. doi:10.1111/joim.12021

34. Aoki M, Burchill P, Yates B, Golding JF (2000) Gresty MA Graviceptive control of blood pressure in man. Arch Ital Biol 138(1):93-97

35. Hammam E, Kwok K, Macefield VG (2013) Modulation of muscle sympathetic nerve activity by low-frequency physiological activation of the vestibular utricle in awake humans. Exp Brain Res 230(1):137-142. doi:10.1007/s00221-013-3637-7 